\title{
Relay Selection and Subcarrier-Pair Based Energy-Efficient Resource Allocation for Multirelay Cooperative OFDMA Networks
}

\author{
Wanming Hao, ${ }^{1}$ Shouyi Yang, ${ }^{1}$ and Wanliang $\mathrm{Hao}^{2}$ \\ ${ }^{1}$ School of Information Engineering, Zhengzhou University, Zhengzhou 450001, China \\ ${ }^{2}$ College of Navigation and Aerospace Engineering, Information Engineering University, Zhengzhou 450001, China
}

Correspondence should be addressed to Wanming Hao; wmhao@hotmail.com

Received 8 May 2014; Revised 13 June 2014; Accepted 13 June 2014; Published 10 July 2014

Academic Editor: Theodoros Tsiftsis

Copyright (C) 2014 Wanming Hao et al. This is an open access article distributed under the Creative Commons Attribution License, which permits unrestricted use, distribution, and reproduction in any medium, provided the original work is properly cited.

\begin{abstract}
Energy-efficient resource allocation is investigated for a relay-based multiuser cooperation orthogonal frequency division multiple access (OFDMA) uplink system with amplify-and-forward (AF) protocol for all relays. The objective is to maximize the total energy efficiency (EE) of the uplink system with consideration of some practical limitations, such as the individual power constraint for the users and relays and the quality of service (QoS) for every user. We formulate an energy-efficient resource allocation problem that seeks joint optimization of subcarrier pairing, relay selection, subcarrier assignment, and power allocation. Unlike previous optimization throughput models, we transform the considered EE problem in fractional form into an equivalent optimal problem in subtractive form, which is solved by using dual decomposition and subgradient methods. To reduce computation costs, we propose two low-complexity suboptimal schemes. Numerical studies are conducted to evaluate the EE of the proposed algorithms.
\end{abstract}

\section{Introduction}

Transmit diversity generally requires more than one antenna at the transmitter and receiver. However, many wireless devices are limited to single antenna because of size or hardware complexity. Cooperation communication is a promising solution to address this problem in various wireless systems, such as ad hoc and cellular networks $[1,2]$. In contrast to traditional point-to-point or point-to-multipoint wireless communication, cooperation communication allows different users or nodes in a wireless system to share resources and create collaboration through distributed transmission; every piece of user's information is sent not only by itself but also by the collaborating users or nodes [3]. Therefore, cooperative communication formulates a new form of space diversity to combat the negative effect of severe fading [4]. Cooperative communication can improve the overall system performance by improving the spectrum efficiency, extending the coverage area, and prolonging the network lifetime. Orthogonal frequency division multiple access (OFDMA) or multiuser orthogonal frequency division multiplexing (MUOFDM) is the preferred multiple access scheme for highspeed wireless multiuser communication networks because of its high spectrum efficiency and resistance to multipath fading [5]. Moreover, several users' signals are simultaneously transmitted over different subcarriers on each OFDM symbol to avoid interference between users. Thus, to improve system performance, studying optimal resource allocation in relayassisted OFDMA communication system is important.

Relaying protocols have three main types, namely, amplify-and-forward (AF), decode-and-forward (DF), and compress-and-forward (CF). In AF, the signal received by relay is amplified and retransmitted to the destination. The noise is also amplified at the relay. This protocol is simple and of low cost. In DF, the relay attempts to decode the received signal. If successful, DF reencodes the information and retransmits the signal. CF attempts to generate an estimate for the received signal. Hoping the estimated value provides some assistance in decoding the original codeword at the destination. Given the limited available space, we only study 
the energy-efficient resource allocation with AF protocol. The other relaying protocols will be studied in future works.

Compared with previous studies on single-carrier relay networks or multicarrier noncooperation networks, more technical challenges exist in the study of multicarrier cooperation networks. We not only consider the relaying protocols (AF, DF, and $\mathrm{CF}$ ) but also solve the problems about relay selection and power allocation between users and relays in relaying networks. Several results have been reported recently about relaying networks [6-14]. The optimal power allocation and subcarrier pairing under AF and DF relay links have been studied, and the optimality of ordered subcarrier pairing (OSP) for these two protocols without diversity under total power constraint has been proved [6]. However, multiple relays usually exist in a practical system; thus, subcarrierto-relay assignment must be considered [8]. The authors in [9] attempted to solve subcarrier-to-relay assignment and subcarrier pairing problems in multirelay OFDM system with a single user. Individual and total power constraints were all considered, but multiple users were not studied. In [10], the authors studied optimal relay selection, power allocation, and subcarrier assignment scheme under total power constraint. However, individual power constraint and subcarrier pairing were not considered. Optimal power and time allocation under a long-term total power constraint in OFDM-based linear multihop relay networks with DF protocol were considered in [11, 12]. In [13], the authors considered that a subcarrier may carry a signal for different services. They also proposed maximizing the weighted sum rate by joint optimal subcarrier pairing and power allocation under total and individual power constraints for the source and relay. Cross-layer scheduling for the downlink of AF relay-assisted OFDMA networks under imperfect channelstate information at the transmitter in slow fading was introduced in [14]. The authors optimized the rate, power, and subcarrier allocation to maximize the system goodput.

We have discussed only the throughput maximum problem in resource allocation for relay-assisted cooperative OFDMA systems. However, explosively growing data traffic and the requirement for ubiquitous access have triggered the escalation of energy, which results in increased greenhouse gas emission [15]. Therefore, energy efficiency (EE) has become an important problem in later development. Besides, mobile terminals cannot connect to an external charger; $\mathrm{EE}$ (bits/Hz/Joule) may be a better performance metric than system throughput (bits/s/Hz) in evaluating the performance of wireless communication systems [1618]. A number of studies on energy-efficient resource allocation have been reported [19-24]. Power adaptation for maximizing the EE in downlink and uplink OFDMA cellular networks was studied in [19]. For downlink transmission, the total EE was maximized; for the uplink case, the minimum individual EE was maximized. In [20], the fundamental tradeoff between $\mathrm{EE}$ and spectral efficiency (SE) in downlink OFDMA systems was studied. In [21], the authors transformed the considered EE problem in fractional form into an equivalent optimal problem in subtractive form. They also studied the resource allocation for energy-efficient communication in multicell OFDMA downlink networks. References [22, 23] used the Dinkelbach method to solve the EE maximum problem in OFDM systems and found the optimal resource allocation. The literature in [24] used the resembled solution in [17] to deal with the energy-efficient resource allocation problem in a multiuser OFDMA system. Users' quality-of-service (QoS) requirements were also considered.

For relaying networks, limited study about EE exists. The authors in [25] formulated EE optimization problem by joint subcarrier assignment, bit, and power allocation. However, they provided only near-optimal and suboptimal resource schemes to maximize the overall $\mathrm{EE}$ and did not find the global optimum. Power constraints for sources and relay were also not considered. The power allocation to maximize EE for a two-hop AF relay link under QoS requirement has also been investigated [26]; single user and relay have been studied, but the subcarrier pairing problem has not been considered.

Considerable research exists about the optimal resource allocation mechanisms in relaying networks, including power allocation, subcarrier pairing, and relay selection; see, for example, [6-14]. However, these problems have only been studied in the case of throughput maximization (supposing throughput as objective function). Optimal resource allocation mechanisms will be changed when we consider the system EE (supposing EE as objective function). Hence, we must provide a new optimal resource allocation mechanism to meet the requirements of systems. In this paper, we address the energy-efficient resource allocation in AF-based cooperative two-hop multirelay uplink OFDMA system. Considering an actual situation, individual power constraint for the users and relays is applied. QoS for every user should be considered. Then, the subcarrier pairing, relay selection, subcarrier assignment, and power allocation problem are formulated as a joint optimization problem with the objective of maximizing the uplink system total EE. Given integer assignment variables, the optimal EE problem falls within the scope of combinatorial programming, which is NPhard. Thus, we transform the EE problem in fractional form into an equivalent optimal problem in subtractive form, which is solved by using dual decomposition and subgradient methods. We also propose two suboptimal algorithms to trade off performance and complexity.

The remainder of this paper is organized as follows. Section 2 introduces the system model and formulates the optimization EE problem. Section 3 presents the optimal resource allocation algorithm. Section 4 shows suboptimal algorithms with low complexity. Section 5 demonstrates simulation results to verify the performance of the proposed algorithms. Section 6 concludes the paper.

\section{System Model and Problem Formulation}

We consider a multirelay-assisted cooperation OFDMA system, as shown in Figure 1. $K$ users $(1 \leq k \leq K)$ and $N$ relays $(1 \leq n \leq N)$ are assumed. The relays are shared by all users. The total number of the subcarriers used is $M$ $(1 \leq m \leq M)$ in the single cell system. Broadband channel is assumed to be frequency-selective Rayleigh fading. All 


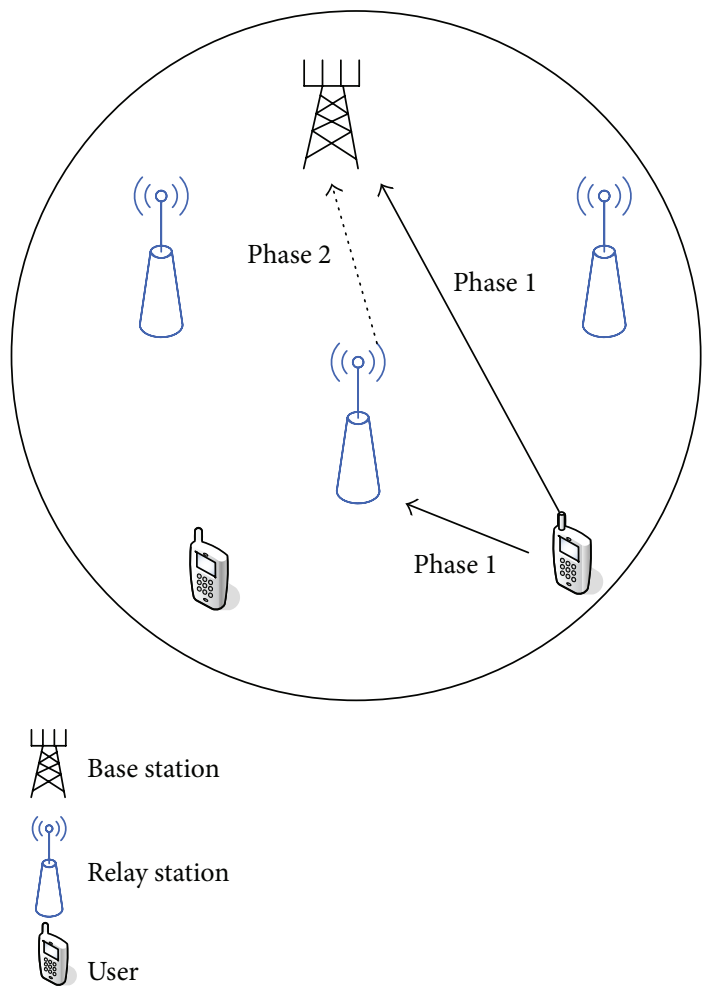

FIGURE 1: System model.

channel-state information is assumed to be perfectly known. Each relay node operates in a time-division half-duplex mode with AF protocol. Therefore, two phases are needed for the communication between the user and base station. In the first phase, the user transmits the signal to the base station, which is overheard by the selected relay as well. In the second phase, the selected relay forwards the received signal to the base station using AF cooperation protocol. $n$ is supposed to receive the signal from user $k$ on subcarrier $m$ in the first phase, and the signal is then forwarded on subcarrier $m^{\prime}$ in the second phase; the subcarrier $m$ may not be the same as $m^{\prime}$, and they are thus called a subcarrier pair $\left(m, m^{\prime}\right)[9]$.

The noise variances of the source-to-relay (SR) links, relay-to-destination (RD) links, and source-to-destination (SD) links are denoted by $\sigma_{k, n}^{2}, \sigma_{n, D}^{2}$, and $\sigma_{k, D}^{2}$, respectively. The channel coefficients of $S \rightarrow R$ and $S \rightarrow D$ links on the subcarrier $m$ in the first phase are denoted by $h_{k, n}^{m}$ and $h_{k, D}^{m}$, respectively, and that of the $R \rightarrow D$ links on the subcarrier $m^{\prime}$ in the second phase is denoted by $h_{n, D}^{m^{\prime}}$. The efficient channel gain of the three links on the subcarrier $m$ or $m^{\prime}$ for user $k$ and relay $n$ can be denoted by $\alpha_{k, n}^{m}=\left|h_{k, n}^{m}\right|^{2} / \sigma_{k, n}^{2}$, $\alpha_{k, D}^{m}=\left|h_{k, D}^{m}\right|^{2} / \sigma_{k, D}^{2}$, and $\alpha_{n, D}^{m^{\prime}}=\left|h_{n, D}^{m^{\prime}}\right|^{2} / \sigma_{n, D}^{2}$, respectively. The channel variances of $S \rightarrow R, S \rightarrow D$, and $R \rightarrow D$ links are denoted by $\delta_{k, n}^{2}, \delta_{k, D}^{2}$, and $\delta_{n, D}^{2}$, respectively.

In this relaying system, the base station controls all users and relays. The resource allocation information, including subcarrier pairing, relay selection, subcarrier assignment, and power allocation, is sent to users and relays by the base station via downlink control channel.
The achievable rate for the $k$ th user in the subcarrier pair $\left(m, m^{\prime}\right)$ when the $n$th relay is selected is given by

$$
r_{k, n}^{m, m^{\prime}}=\frac{1}{2} \log _{2}\left(1+p_{S, k}^{m} \alpha_{k, D}^{m}+\frac{p_{S, k}^{m} \alpha_{k, n}^{m} p_{R, n}^{m^{\prime}} \alpha_{n, D}^{m^{\prime}}}{1+p_{S, k}^{m} \alpha_{k, n}^{m}+p_{R, n}^{m^{\prime}} \alpha_{n, D}^{m^{\prime}}}\right),
$$

where $p_{S, k}^{m}$ is the transmit power of the $k$ th user in the $m$ th subcarrier and $p_{R, n}^{m^{\prime}}$ is the transmit power of the $n$th relay in the $m^{\prime}$ th subcarrier. The factor $1 / 2$ is due to the two-phase transmission.

$r_{k, n}^{m, m^{\prime}}$ is not jointly concave in $p_{S, k}^{m}$ and $p_{R, n}^{m^{\prime}}$. To make the problem tractable, we adopt the following approximation:

$$
r_{k, n}^{m, m^{\prime}} \approx \frac{1}{2} \log _{2}\left(1+p_{S, k}^{m} \alpha_{k, D}^{m}+\frac{p_{S, k}^{m} \alpha_{k, n}^{m} p_{R, n}^{m^{\prime}} \alpha_{n, D}^{m^{\prime}}}{p_{S, k}^{m} \alpha_{k, n}^{m}+p_{R, n}^{m^{\prime}} \alpha_{n, D}^{m^{\prime}}}\right) .
$$

The above approximation is jointly concave in $p_{S, k}^{m}$ and $p_{R, n}^{m^{\prime}}$, as proved in Appendix A. This approximation is based on the assumption that the signal is amplified and forwarded by the relay under a high signal-to-ratio (SNR) condition. Such approximation has been used in the literatures $[7,9$, 27]. However, in a low-SNR regime, [27] proved that the resource allocation almost reaches the true optimal capacity by optimizing the rate (2).

Our objective is to maximize the uplink system total EE subject to a set of constraints. The relay selection and subcarrier assignment constraints are as follows:

$$
\sum_{k=1}^{K} \sum_{n=1}^{N} \rho_{k, n}^{m, m^{\prime}}=1, \quad \rho_{k, n}^{m, m^{\prime}} \in\{0,1\}, \forall m, m^{\prime},
$$

where $\rho_{k, n}^{m, m^{\prime}}=1$ indicates that user $n$ uses relay $k$ as a relay in the subcarrier pair $\left(m, m^{\prime}\right)$; otherwise, $\rho_{k, n}^{m, m^{\prime}}=0$.

$\gamma_{m, m^{\prime}}$ denotes the indicator for subcarrier pairing. When $\gamma_{m, m^{\prime}}=1$, subcarrier $m$ in the first phase is paired with subcarrier $m^{\prime}$ in the second phase; otherwise, $\gamma_{m, m^{\prime}}=0$. Given that each subcarrier in the first phase can be paired with only one subcarrier in the second phase, the binary variables $\left\{\gamma_{m, m^{\prime}}\right\}$ must satisfy the following equation:

$$
\sum_{m=1}^{M} \gamma_{m, m^{\prime}}=1, \quad \sum_{m^{\prime}=1}^{M} \gamma_{m, m^{\prime}}=1, \quad \forall m, m^{\prime} .
$$

The individual power constraint of the users and relays can be expressed as follows:

$$
\begin{gathered}
\text { User power constraint: } \sum_{m=1}^{M} p_{S, k}^{m} \leq P_{S, k}, \quad \forall k, \\
\text { Relay power constraint: } \sum_{m^{\prime}=1}^{M} p_{R, n}^{m^{\prime}} \leq P_{R, n}, \quad \forall n .
\end{gathered}
$$

Aside from transmit power, the energy consumption also includes circuit energy consumption [28] and overall power 
consumption. The power consumptions of user $n$ and relay $k$ are modeled as follows:

user power consumption: $P_{S, k}^{\text {total }}=\xi \sum_{m=1}^{M} p_{S, k}^{m}+P_{C, S}^{k}$,

relay power consumption: $P_{R, n}^{\text {total }}=\xi \sum_{m^{\prime}=1}^{M} p_{R, n}^{m^{\prime}}+P_{C, R}^{n}$,

where $\xi$ is the reciprocal of drain efficiency of power amplifier. We assume that the reciprocal is the same for all the users and relays.

The optimization EE problem can be formulated as

$$
\begin{aligned}
\max \mathrm{EE}= & \left(\sum_{k=1}^{K} w_{k} \sum_{n=1}^{N} \sum_{m=1}^{M} \sum_{m^{\prime}=1}^{M} \rho_{k, n}^{m, m^{\prime}} \gamma_{m, m^{\prime}} r_{k, n}^{m, m^{\prime}}\right) \\
& \times\left(\xi \sum_{k=1}^{K} \sum_{n=1}^{N} \sum_{m=1}^{M} \sum_{m^{\prime}=1}^{M} \rho_{k, n}^{m, m^{\prime}} \gamma_{m, m^{\prime}}\left(p_{S, k}^{m}+p_{R, n}^{m^{\prime}}\right)\right. \\
& \left.+\sum_{k=1}^{K} P_{C, S}^{k}+\sum_{n=1}^{N} P_{C, R}^{n}\right)^{-1},
\end{aligned}
$$

which is subject to

$$
\begin{gathered}
\rho_{k, n}^{m, m^{\prime}} \in\{0,1\}, \quad \forall k, n, m, m^{\prime}, \\
\sum_{k=1}^{K} \sum_{n=1}^{N} \rho_{k, n}^{m, m^{\prime}}=1, \quad \forall m, m^{\prime}, \\
\gamma_{m, m^{\prime}} \in\{0,1\}, \quad \forall m, m^{\prime}, \\
\sum_{m=1}^{M} \gamma_{m, m^{\prime}}=1, \quad \sum_{m^{\prime}=1}^{M} \gamma_{m, m^{\prime}}=1, \quad \forall m, m^{\prime}, \\
\sum_{m=1}^{M} p_{S, k}^{m} \leq P_{S, k}, \quad \forall k, \\
\sum_{m^{\prime}=1}^{M} p_{R, n}^{m^{\prime}} \leq P_{S, n}, \quad \forall n, \\
R_{S, k} \geq Q_{S, k}, \quad \forall k, \\
p_{S, k}^{m} \geq 0, \quad p_{R, n}^{m^{\prime}} \geq 0, \quad \forall k, n, m, m^{\prime},
\end{gathered}
$$

where constraint (14) denotes the minimum QoS requirements for the users and $R_{S, k}=\sum_{n=1}^{N} \sum_{m=1}^{M} \sum_{m^{\prime}=1}^{M} r_{k, n}^{m, m^{\prime}}$. The predetermined weights, $w_{k}$, can provide a certain level of priority and/or fairness among users.

\section{Optimal Resource Allocation}

The integer assignment variables $\rho_{k, n}^{m, m^{\prime}}$ and $\gamma_{m, m^{\prime}}$ above EE problem are generally NP-hard for optimal solution. If we consider all possibilities of subcarrier pairings and subcarrier-pair-to-user and relay assignments, then the complexity is high with large $K, N$, and $M$. The fraction further complicates the problem. Hence, we apply an effective method to solve this challenging problem.

3.1. Equivalence Transformation of the Optimization Problem. For the fraction objective function in (7), we first rewrite problems (7)-(15) in the following compact form:

$$
\underset{\boldsymbol{\rho}, \boldsymbol{\gamma}, \mathbf{p} \in \pi}{\operatorname{maximize}} \frac{R(\boldsymbol{\rho}, \boldsymbol{\gamma}, \mathbf{p})}{P(\boldsymbol{\rho}, \boldsymbol{\gamma}, \mathbf{p})},
$$

where $\boldsymbol{\rho}=\left\{p_{k, n}^{m, m^{\prime}}\right\}, \boldsymbol{\gamma}=\left\{\gamma_{m, m^{\prime}}\right\}$, and $\mathbf{p}=\left\{p_{S, k}^{m}, p_{R, n}^{m^{\prime}}\right\} . \boldsymbol{\pi}$ denotes the feasible domain defined by (8)-(15). $R(\boldsymbol{\rho}, \boldsymbol{\gamma}, \mathbf{p})$ and $P(\boldsymbol{\rho}, \boldsymbol{\gamma}, \mathbf{p})$ are the numerator and denominator in (7), respectively. Problem (16) is a nonlinear fractional problem. According to [29], we transform the considered EE problem in fractional form into an equivalent optimal problem in subtractive form. We then define the following parameter problem:

$$
F(q) \triangleq \operatorname{maximize}_{\boldsymbol{\rho}, \boldsymbol{\gamma}, \mathbf{p} \in \boldsymbol{\pi}}\{R(\boldsymbol{\rho}, \boldsymbol{\gamma}, \mathbf{p})-q P(\boldsymbol{\rho}, \boldsymbol{\gamma}, \mathbf{p})\},
$$

where $q \in \mathbb{R}$ is treated as a parameter. We have the following theorems, all of which are proved in Appendix B.

Theorem 1. $F(q)$ is strictly monotonically decreasing for $q$.

Theorem 2. $F(q)=0$ has a unique solution.

Theorem 3. Consider

$$
\begin{aligned}
& q_{0}=\frac{R\left(\boldsymbol{\rho}_{0}, \boldsymbol{\gamma}_{0}, \mathbf{p}_{0}\right)}{P\left(\boldsymbol{\rho}_{0}, \boldsymbol{\gamma}_{0}, \mathbf{p}_{0}\right)}=\max _{\boldsymbol{\rho}, \boldsymbol{\gamma}, \mathbf{p} \in \pi} \frac{R(\boldsymbol{\rho}, \boldsymbol{\gamma}, \mathbf{p})}{P(\boldsymbol{\rho}, \boldsymbol{\gamma}, \mathbf{p})} \quad \text { only if } \\
& \begin{aligned}
F\left(q_{0}\right) & =F\left(q_{0},\left(\boldsymbol{\rho}_{0}, \boldsymbol{\gamma}_{0}, \mathbf{p}_{0}\right)\right) \\
& =\max _{\boldsymbol{\rho}, \boldsymbol{\gamma}, \mathbf{p} \in \boldsymbol{\pi}}\left\{R(\boldsymbol{\rho}, \boldsymbol{\gamma}, \mathbf{p})-q_{0}(\boldsymbol{\rho}, \boldsymbol{\gamma}, \mathbf{p})\right\}=0 .
\end{aligned}
\end{aligned}
$$

Based on the above theorems, we can use binary search method to find $F(q)=0$ and the optimal solution for the primal problem. Therefore, the difficulty is to solve $T(q)$ for a given $q$. The optimization problem is

$$
\max _{\boldsymbol{\rho}, \boldsymbol{\gamma}, \mathbf{p} \in \boldsymbol{\pi}} R(\boldsymbol{\rho}, \boldsymbol{\gamma}, \mathbf{p})-q(\boldsymbol{\rho}, \boldsymbol{\gamma}, \mathbf{p}) .
$$


Obtaining the joint optimal $\boldsymbol{\rho}^{*}, \boldsymbol{\gamma}^{*}$, and $\mathbf{p}^{*}$ for (19) requires solving a mixed integer programming problem. Therefore, finding the optimal solution for problem (19) requires searching through all possible users, relays, and subcarrier allocation, which is a complex task when the system is large. The literature in [30] showed that the duality gap of a nonconvex resource allocation problem that satisfies the time-sharing condition is nearly zero with a large number of subcarriers in a multicarrier system. Thus, we solve this problem by using the Lagrange dual method.

3.2. Dual Problem. The Lagrange dual function of problem (19) can be written as

$$
g(\boldsymbol{\lambda}, \boldsymbol{\mu}, \boldsymbol{v})=\max _{\{\boldsymbol{\rho}, \boldsymbol{\gamma}\} \in \Omega} L(\boldsymbol{\rho}, \boldsymbol{\gamma}, \mathbf{p}, \boldsymbol{\lambda}, \boldsymbol{\mu}, \boldsymbol{v}),
$$

$$
\begin{aligned}
& L(\boldsymbol{\rho}, \boldsymbol{\gamma}, \mathbf{p}, \boldsymbol{\lambda}, \boldsymbol{\mu}, \boldsymbol{v}) \\
& =\sum_{k=1}^{K} \sum_{n=1}^{N} \sum_{m=1}^{M} \sum_{m^{\prime}=1}^{M} \frac{w_{k}}{2} \rho_{k, n}^{m, m^{\prime}} \gamma_{m, m^{\prime}} \\
& \quad \times \log _{2}\left(1+p_{S, k}^{m} \alpha_{k, D}^{m}+\frac{p_{S, k}^{m} \alpha_{k, n}^{m} p_{R, n}^{m^{\prime}} \alpha_{n, D}^{m^{\prime}}}{p_{S, k}^{m} \alpha_{k, n}^{m}+p_{R, n}^{m^{\prime}} \alpha_{n, D}^{m^{\prime}}}\right) \\
& -q\left(\xi \sum_{k=1}^{K} \sum_{n=1}^{N} \sum_{m=1}^{M} \sum_{m^{\prime}=1}^{M} \rho_{k, n}^{m, m^{\prime}} \gamma_{m, m^{\prime}}\left(p_{S, k}^{m}+p_{R, n}^{m^{\prime}}\right)\right. \\
& \left.+\sum_{k=1}^{K} P_{C, S}^{k}+\sum_{n=1}^{N} P_{C, R}^{n}\right)+\sum_{k=1}^{k} \lambda_{k}\left(P_{S, k}-\sum_{m=1}^{M} p_{S, k}^{m}\right) \\
& +\sum_{n=1}^{N} \mu_{n}\left(P_{S, n}-\sum_{m^{\prime}=1}^{M} p_{R, n}^{m^{\prime}}\right)+\sum_{k=1}^{k} v_{k}\left(R_{S, k}-Q_{S, k}\right),
\end{aligned}
$$

where Lagrange is given in (21).

In $(20), \boldsymbol{\lambda}=\left(\lambda_{1}, \lambda_{2}, \ldots, \lambda_{K}\right) \geq 0, \boldsymbol{\mu}=\left(\mu_{1}, \mu_{2}, \ldots, \mu_{N}\right) \geq$ 0 , and $\boldsymbol{v}=\left(v_{1}, v_{2}, \ldots, v_{K}\right) \geq 0$ are the vectors of the dual variables associated with the individual power constraint for the users, individual power constraint for the relays, and individual rate constraint for the users, respectively. Hence, the dual optimization problem is given by

$$
\begin{aligned}
\min & g(\boldsymbol{\lambda}, \boldsymbol{\mu}, \boldsymbol{v}), \\
\text { s.t. } & \boldsymbol{\lambda}, \boldsymbol{\mu}, \boldsymbol{v} \geq 0 .
\end{aligned}
$$

The literature in [31] proved that a dual function is always convex. Therefore, subgradient-based methods can be used to minimize $g(\boldsymbol{\lambda}, \boldsymbol{\mu}, \boldsymbol{v})$ with guaranteed convergence. Dual variables can be updated in parallel as follows:

$$
\begin{gathered}
\lambda_{k}(d+1)=\left[\lambda_{k}(d)+o(d)\left(\sum_{m=1}^{M} p_{S, k}^{m}-P_{S, k}\right)\right]^{+} \\
\mu_{n}(d+1)=\left[\mu_{n}(d)+\theta(d)\left(\sum_{m^{\prime}=1}^{M} p_{R, n}^{m^{\prime}}-P_{S, n}\right)\right]^{+} \\
v_{k}(d+1)=\left[v_{k}(d)+\vartheta(d)\left(Q_{S, k}-R_{S, k}\right)\right]^{+} .
\end{gathered}
$$

$o(d), \theta(d)$, and $\vartheta(d)$ are used as the step size, and $d$ is the iteration index. To guarantee optimal dual variable convergence, the step size is chosen following the diminishing step size policy [32].

3.3. Optimizing Primal Variables with Given Dual Variables. Computing the dual function $g(\boldsymbol{\lambda}, \boldsymbol{\mu}, \boldsymbol{v})$ involves determining the optimal $\rho, \gamma$, and $\mathbf{p}$ at given dual points $\lambda, \mu$, and $\boldsymbol{v}$. Therefore, we present the detailed solution procedure in three phases.

3.3.1. Finding the Optimal $\mathbf{p}$ for Fixed $\boldsymbol{\rho}$ and $\boldsymbol{\gamma}$. For the fixed $\rho$ and $\gamma$, we rewrite (20) as

$$
\begin{aligned}
g(\boldsymbol{\lambda}, \boldsymbol{\mu}, \boldsymbol{v})= & \max _{\{\boldsymbol{\rho}, \boldsymbol{\gamma}\} \in \boldsymbol{\Omega}} \sum_{k=1}^{K} \sum_{n=1}^{N} \sum_{m=1}^{M} \sum_{m^{\prime}=1}^{M} L_{k, n}^{m, m^{\prime}}+\sum_{k=1}^{k} \lambda_{k} P_{S, k} \\
& +\sum_{n=1}^{N} \mu_{n} P_{S, n}+q\left(\sum_{k=1}^{K} P_{C, S}^{k}+\sum_{n=1}^{N} P_{C, R}^{n}\right)-\sum_{k=1}^{k} v_{k} Q_{S, k},
\end{aligned}
$$

where

$$
\begin{aligned}
L_{k, n}^{m, m^{\prime}} \triangleq & \frac{w_{k}}{2}\left(1+v_{k}\right) \\
& \times \log _{2}\left(1+p_{S, k}^{m} \alpha_{k, D}^{m}+\frac{p_{S, k}^{m} \alpha_{k, n}^{m} p_{R, n}^{m^{\prime}} \alpha_{n, D}^{m^{\prime}}}{p_{S, k}^{m} \alpha_{k, n}^{m}+p_{R, n}^{m^{\prime}} \alpha_{n, D}^{m^{\prime}}}\right) \\
& -\left(q \xi+\lambda_{k}\right) p_{S, k}^{m}-\left(q \xi+\mu_{n}\right) p_{R, n}^{m^{\prime}} .
\end{aligned}
$$

A detailed derivation is given in Appendix C.

According to the Lagrange dual decomposition method, the optimal power allocation can be determined by solving the following problem:

$$
\begin{gathered}
\max L_{k, n}^{m, m^{\prime}} \\
p_{S, k}^{m} \geq 0, \quad p_{R, n}^{m^{\prime}} \geq 0, \quad \forall k, n, m, m^{\prime} .
\end{gathered}
$$

Appendix A indicates that $L_{k, n}^{m, m^{\prime}}$ is a joint concave function of $\left(p_{S, k}^{m}, p_{R, n}^{m^{\prime}}\right)$. Applying the Karush-Kuhn-Tucker (KKT) conditions [28], we can obtain the following optimal power allocation: 


$$
\begin{aligned}
& p_{S, k}^{m}= \begin{cases}b_{k, n}^{m, m^{\prime}} p_{R, n}^{m^{\prime}}, & \text { if } p_{R, n}^{m^{\prime}}>0 \\
\left(\frac{w_{k}\left(1+v_{k}\right)}{2 \ln 2\left(q \xi+\lambda_{k}\right)}-\frac{1}{\alpha_{k, D}^{m}}\right)^{+} & \text {if } p_{R, n}^{m^{\prime}}=0\end{cases} \\
& p_{R, n}^{m^{\prime}}=\left\{\begin{array}{c}
\left(\begin{array}{c}
\frac{w_{k}\left(1+v_{k}\right)}{2 \ln 2}\left(\alpha_{k, n}^{m} \alpha_{n, D}^{m^{\prime}}{ }^{2}+\left(\alpha_{k, D}^{m}-\left(2\left(q \xi+\lambda_{k}\right) \ln 2\right) /\left(w_{k}\left(1+v_{k}\right)\right)\right)\right. \\
\left.\times\left(\alpha_{k, n}^{m} b_{k, n}^{m, m^{\prime}}+\alpha_{n, D}^{m^{\prime}}\right)^{2}\right) \\
\times\left(b_{k, n}^{m, m^{\prime}}\left(q \xi+\lambda_{k}\right)\left(\alpha_{k, n}^{m} b_{k, n}^{m, m^{\prime}}+\alpha_{n, D}^{m^{\prime}}\right)\right. \\
\left.\times\left(\alpha_{k, D}^{m} \alpha_{k, n}^{m} b_{k, n}^{m, m^{\prime}}+\alpha_{n, D}^{m^{\prime}} \alpha_{k, D}^{m}+\alpha_{k, n}^{m} \alpha_{n, D}^{m^{\prime}}\right)\right)^{-1}
\end{array}\right)+\lambda_{k, D}^{+}\left(q \xi+\mu_{n}\right) \\
\text { if } \alpha_{n, D}^{m^{\prime}}\left(q \xi+\lambda_{k}\right)>\alpha_{k, D}^{m}\left(q \xi+\lambda_{k}\right) \leq \alpha_{k, D}^{m}\left(q \xi+\mu_{n}\right) . \\
\text { if } \alpha_{n, D}^{m^{\prime}}(q \xi
\end{array}\right. \\
& b_{k, n}^{m, m^{\prime}}=\frac{\alpha_{n, D}^{m^{\prime}}\left(\sqrt{\left(q \xi+\mu_{n}\right)\left(\alpha_{k, n}^{m} \alpha_{n, D}^{m^{\prime}}\left(q \xi+\lambda_{k}\right)-\alpha_{k, n}^{m} \alpha_{k, D}^{m}\left(q \xi+\mu_{n}\right)+\alpha_{n, D}^{m^{\prime}} \alpha_{k, D}^{m}\left(q \xi+\lambda_{k}\right)\right)}+\alpha_{k, D}^{m}\left(q \xi+\mu_{n}\right)\right)}{\alpha_{k, n}^{m}\left[\alpha_{n, D}^{m^{\prime}}\left(q \xi+\lambda_{k}\right)-\alpha_{k, D}^{m}\left(q \xi+\mu_{n}\right)\right]} .
\end{aligned}
$$

And $p_{R, n}^{m^{\prime}}$ is given in (28).

A detailed derivation is given in Appendix D.

3.3.2. Finding the Optimal $\rho$ for Fixed $\gamma$. Substituting the optimal power allocation expressions (27) and (28) into (20), we can obtain an alternative expression of the dual without power variables as

$$
\begin{aligned}
g(\boldsymbol{\lambda}, \boldsymbol{\mu}, \boldsymbol{v})= & \max _{\{\boldsymbol{\rho}, \gamma\} \in \boldsymbol{\Omega}} \sum_{k=1}^{K} \sum_{n=1}^{N} \sum_{m=1}^{M} \sum_{m^{\prime}=1}^{M} \rho_{k, n}^{m, m^{\prime}} \gamma_{m, m^{\prime}} H_{k, n}^{m, m^{\prime}}(\boldsymbol{\lambda}, \boldsymbol{\mu}, \boldsymbol{v}) \\
& +\sum_{k=1}^{k} \lambda_{k} P_{S, k}+\sum_{n=1}^{N} \mu_{n} P_{S, n} \\
& +q\left(\sum_{k=1}^{K} P_{C, S}^{k}+\sum_{n=1}^{N} P_{C, R}^{n}\right)-\sum_{k=1}^{k} v_{k} Q_{S, k},
\end{aligned}
$$

where the function $H_{k, n}^{m, m^{\prime}}(\boldsymbol{\lambda}, \boldsymbol{\mu}, \boldsymbol{v})$ can be defined as follows:

$$
H_{k, n}^{m, m^{\prime}}=w_{k}\left(1+v_{k}\right) r_{k, n}^{m, m^{\prime}}-\left(q \xi+\lambda_{k}\right) p_{S, k}^{m}-\left(q \xi+\mu_{n}\right) p_{R, n}^{m^{\prime}} .
$$

Therefore, the function $H_{k, n}^{m, m^{\prime}}(\boldsymbol{\lambda}, \boldsymbol{\mu}, \boldsymbol{v})$ is the optimal criterion for relay and user selection. We can interpret this criterion by using an economic form. The first term can be viewed as the rate obtained by selecting subcarrier pairing $\left(m, m^{\prime}\right)$ by user $k$ and relay $n$, such as our gross income in the business. The second term is the cost for the user's power consumption. The third term is the cost for the relay's power consumption. $H_{k, n}^{m, m^{\prime}}(\boldsymbol{\lambda}, \boldsymbol{\mu}, \boldsymbol{v})$ then represents our net profit. We should find the optimal $k$ and $n$ to maximize the profit. $K \times N$ profit matrix exists for every subcarrier pairing $(m$, $m^{\prime}$ ). We should find a maximum value in this matrix; thus, the sum of profit could be large. The optimal relay selection and subcarrier allocation should therefore be the maximum value in (31) and is given by

$$
\rho_{k, n}^{m, m^{\prime}}= \begin{cases}1, & \left(k^{*}, n^{*}\right)=\arg \max _{k, n} H_{k, n}^{m, m^{\prime}} \\ 0, & \text { otherwise. }\end{cases}
$$

In the operation, the optimal power allocation can first be computed by using (28) and (29). These power values can then be substituted into (31) to compute $H_{k, n}^{m, m^{\prime}}$. Finally, we need to find a maximum value in $K \times N$ profit matrix, and the user and relay pair will be determined.

3.3.3. Finding the Optimal $\gamma$ for Fixed $\rho$. After finding the optimal $\rho$, we can obtain the corresponding dual function by substituting (32) into (20) as follows:

$$
\begin{aligned}
g(\boldsymbol{\lambda}, \boldsymbol{\mu}, \boldsymbol{v})= & \max _{\boldsymbol{\gamma} \in \boldsymbol{\Omega}} \sum_{m=1}^{M} \sum_{m^{\prime}=1}^{M} \gamma_{m, m^{\prime}} H_{m, m^{\prime}}(\boldsymbol{\lambda}, \boldsymbol{\mu}, \boldsymbol{v})+\sum_{k=1}^{k} \lambda_{k} P_{S, k} \\
& +\sum_{n=1}^{N} \mu_{n} P_{S, n}+q\left(\sum_{k=1}^{K} P_{C, S}^{k}+\sum_{n=1}^{N} P_{C, R}^{n}\right)-\sum_{k=1}^{k} v_{k} Q_{S, k} .
\end{aligned}
$$




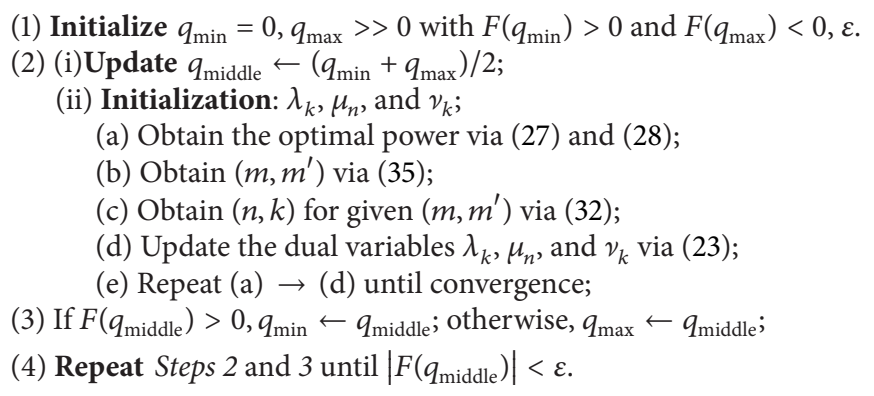

Algorithm 1: Bisection-based energy-efficient resource allocation algorithm.

We can then find the optimal subcarrier pairing $\gamma$. To this end, we launch the first term in (33) and define $M \times M$ profit matrix as follows:

$$
\left[\begin{array}{cccc}
\gamma_{1,1} H_{1,1} & \gamma_{1,2} H_{1,2} & \cdots & \gamma_{1, M} H_{1, M} \\
\gamma_{2,1} H_{2,1} & \gamma_{2,2} H_{2,2} & \cdots & \gamma_{2, M} H_{2, M} \\
\vdots & \vdots & \ddots & \vdots \\
\gamma_{M, 1} H_{M, 1} & \gamma_{M, 2} H_{M, 2} & \cdots & \gamma_{M, M} H_{M, M}
\end{array}\right] .
$$

We need to select one element in each row and each column in matrix (34) for the sum of profit to be large. Clearly, the problem is a standard linear assignment and can be solved by using the Hungarian method [33]. $\Psi(m)$ is defined as the subcarrier index in the second phase optimally paired with subcarrier $m$ in the first phase. The optimal subcarrier pairing can be expressed as

$$
\gamma_{m, m^{\prime}}= \begin{cases}1, & m^{\prime}=\psi(m) \\ 0, & \text { otherwise. }\end{cases}
$$

We have obtained the optimal variables $\rho, \gamma$, and $\mathbf{p}$ for given dual variables $\lambda, \boldsymbol{\mu}$, and $\boldsymbol{v}$. We can subsequently obtain the joint optimization problem by updating the dual variables.

$T(q)$ is obtained according to the preceding analysis for a given $q$. However, we should find the optimal $q$ to satisfy $T(q)=0 . q=0$, which yields $T(q)>0$ and $T(q)<0$ with $q \rightarrow \infty$. Thus, we can use binary search method to find the optimal q, as stated in Algorithm 1.

\section{Suboptimal Schemes}

We analyze the complexity of the proposed optimal scheme. For every subcarrier pair, the number of computations needed to perform relay selection is $K N$. Therefore, the complexity at relay selection for all the subcarrier pair is $O(M K N)$. The complexity of the Hungarian method is $O\left(M^{3}\right)$. The complexity of the subgradient method is polynomial in the number of dual variables. Considering the individual power constraint for the users and relays and the individual rate constraint for the users, the number of the dual variables is $(2 K+N)$. Therefore, the overall complexity is $O\left(|2 K+N|^{2} M^{4} K N \cdot \log _{2}(1 / \varepsilon)\right)$. $\varepsilon$ is the accuracy required for the binary search. In this section, we only propose two schemes for the subcarrier pairing.
4.1. Suboptimal Scheme 1: Order Subcarrier Pairing. Similar to the literature in [6], OSP denotes the best $S \rightarrow R$ subcarrier in the first phase with the best $R \rightarrow D$ subcarrier in the secondary phase, pairing the next best $S \rightarrow R$ subcarrier in the first phase with the next best $R \rightarrow D$ subcarrier in the secondary phase until all the subcarriers are paired. The complexity is $O\left(2 M^{2}\right)$ for OSP. When the number of subcarriers is small, the advantage of using OSP is not obvious. By contrast, the advantage is obvious when the number of subcarriers is large.

4.2. Suboptimal Scheme 2: Fixed Subcarrier Pairing. To reduce the complexity of the subcarrier pairing, we let the subcarrier pairing be prefixed, rather than seeking the optimal subcarrier pairing. Similar to the previous suboptimal scheme [33], the subcarrier pairing can be arranged as

$$
\psi(m)=m, \quad \forall m .
$$

Thus, the source and relay use the same subcarrier to transmit and forward a signal in different phases, respectively. We do not need to make the subcarrier pair.

In the two suboptimal schemes, we only reduce the complexity for subcarrier pairing. The dual variables still need to be updated to compute $H_{k, n}^{m, m^{\prime}}$.

\section{Simulation Results}

In this section, simulation results are presented to demonstrate the performance of the three proposed schemes. In the simulation, we consider quasistatic frequency-selective Rayleigh fading channels with a six-tap equal-gain, equalspace delay profile. The delay interval between adjacent taps is equal to the inverse of the OFDM system bandwidth. We consider $M=48$ subcarriers. The bandwidth of each subcarrier is $15 \mathrm{kHz}$. Without loss of generality, we assume that all the noise terms are complex Gaussian random variables with zero mean and variance with 1 . To produce large channel fading between users and destination, we assume that the channel variance of $S \rightarrow D$ link is $-8 \mathrm{~dB}$. Two users have the same minimum rate requirement of $0.5 \mathrm{bps} / \mathrm{Hz}$. For each user and relay, the maximum transmit power and the circuit power are $1 \mathrm{~W}$ and $0.2 \mathrm{~W}$, respectively. For simplicity, we assume that the drain efficiency of power amplifier is 0.35 . 


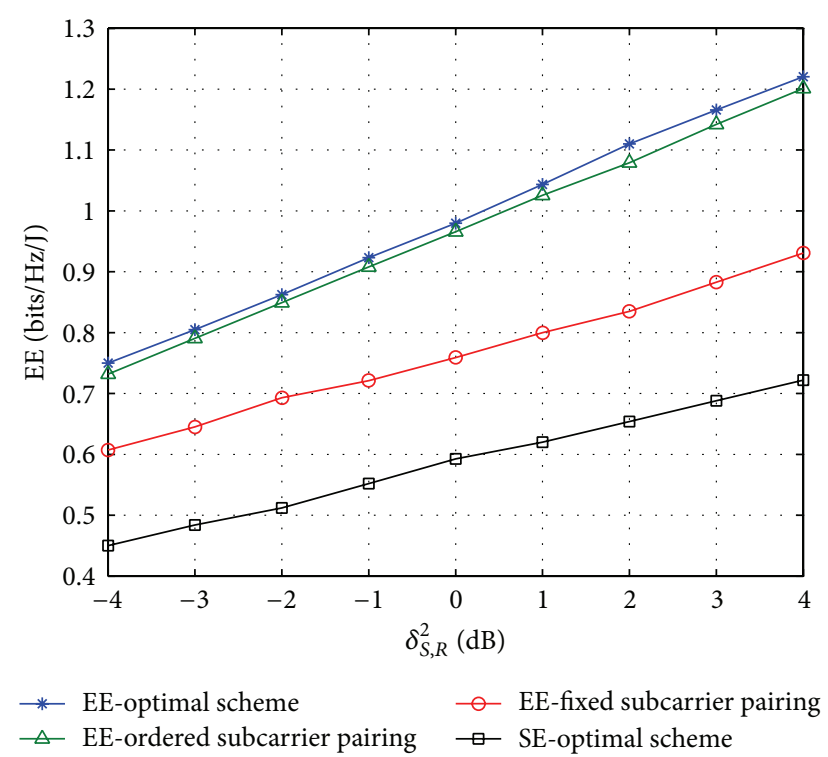

FIGURE 2: Energy efficiency versus channel variance of $S \rightarrow R$ link with $N=2$ relays, and $w_{1}=w_{2}=1$.

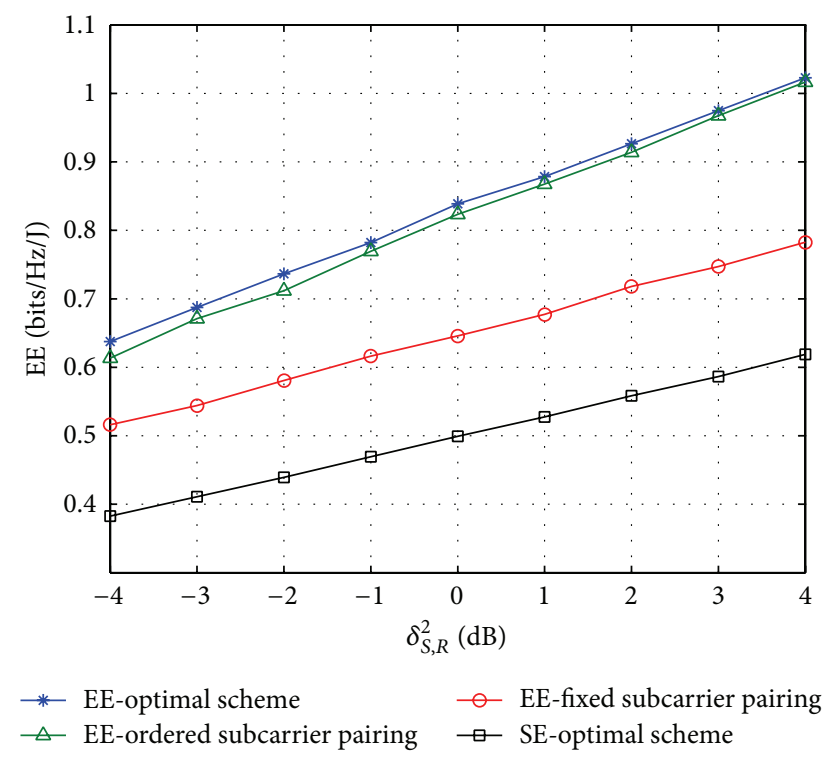

Figure 3: Energy efficiency versus channel variance of $S \rightarrow R$ link with $N=2$ relays, and $w_{1}=1, w_{2}=2$.

EE of the energy-efficient design that optimizes EE and the spectral-efficient design that maximizes the weight sum rate with the same constraints is evaluated in Figures 2 and 3. For any $k$ and $n$, we assume that the value of $\delta_{k, n}^{2}$ is the same and define it as $\delta_{S, R}^{2}$. We set $\delta_{n, D}^{2}=0 \mathrm{~dB}$ for any $n$. Accordingly, the energy-efficient design significantly improves EE compared with the spectral-efficient design. The suboptimal scheme-based OSP is close to the optimal scheme, and its performance reached $97 \%$ or more of the optimal performance. EE performance of the suboptimal scheme-based fixed subcarrier paring is worse compared with the other two schemes. However, this scheme does not need

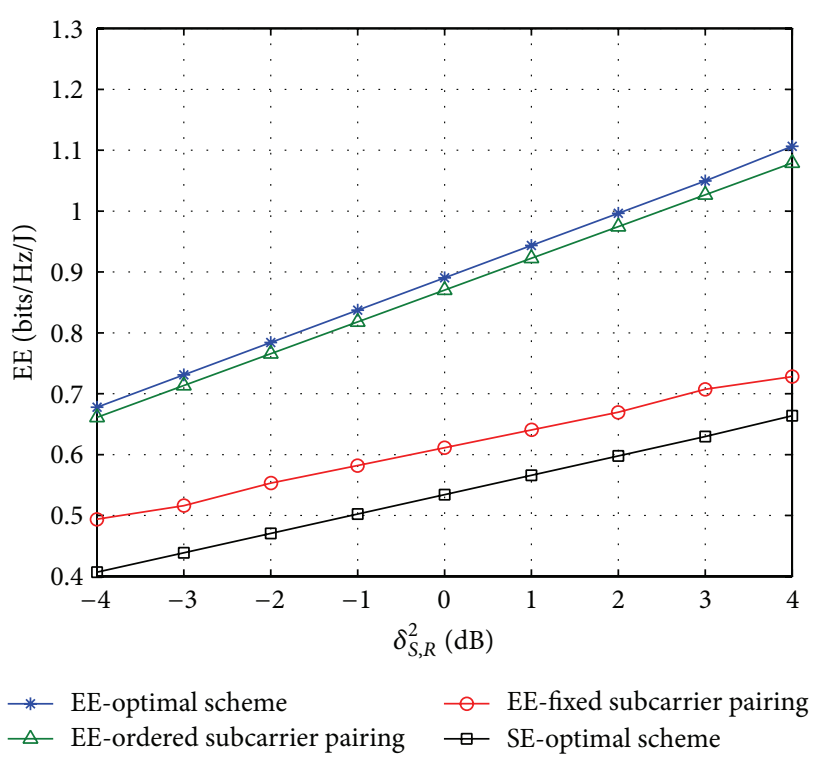

FIGURE 4: Energy efficiency versus channel variance of $S \rightarrow R$ link with $N=12$ relays, and $w_{1}=w_{2}=1$.

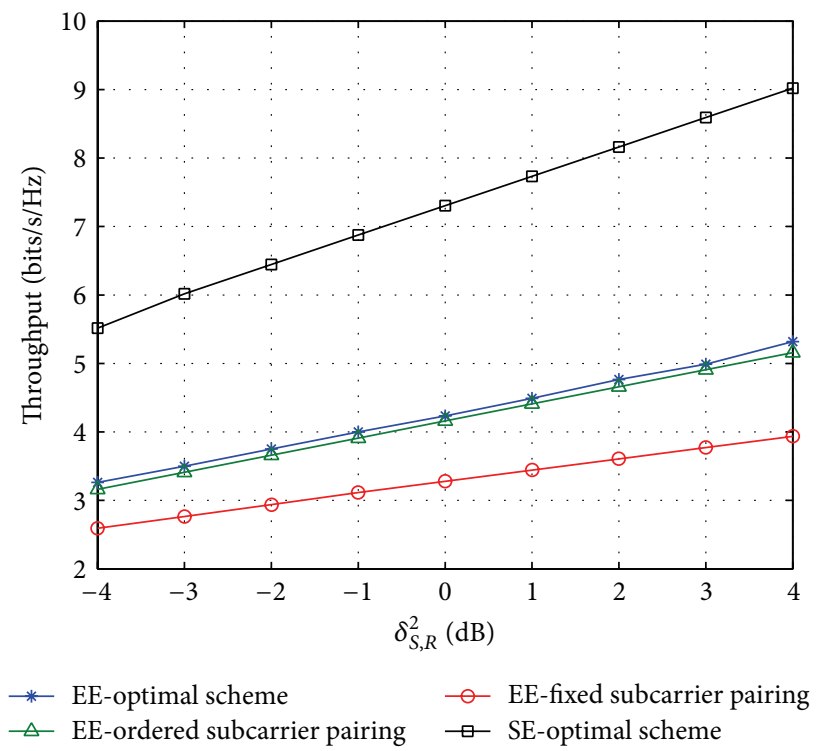

FIgURE 5: Throughput versus channel variance of $S \rightarrow R$ link with $N=2$ relays, and $w_{1}=1, w_{2}=1$.

subcarrier pairing, and its computation complexity is lower. Figure 3 shows that EE will decrease when considering the user's priority, namely, the weight factors.

For comparison with EE performance under different number of relays, we also plot EE with 12 relays with equal weight factor for all the users in Figure 4 . EE in Figure 4 is lower than that in Figure 2 for a given $\delta_{S, R}^{2}$. The literature in [8] indicated that, as the number of relays increases, the average throughput increases. When considering EE, this relationship may be changed, as presented in Figures 2 and 4. Aside from the power consumption of signals, additional circuit energy consumption for the users and relays must be considered. 


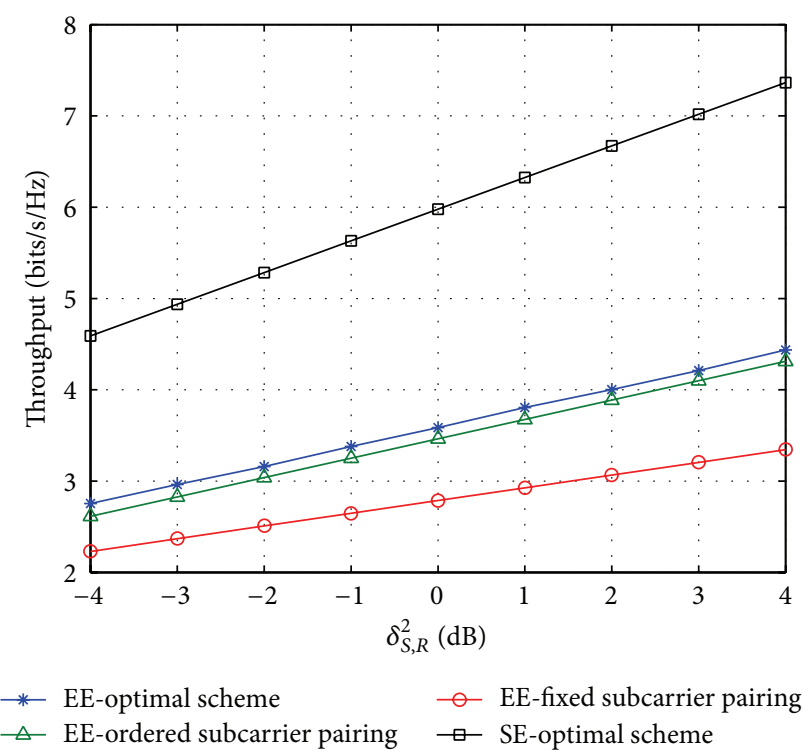

FIgURE 6: Throughput versus channel variance of $S \rightarrow R$ link with $N=2$ relays, and $w_{1}=1, w_{2}=2$.

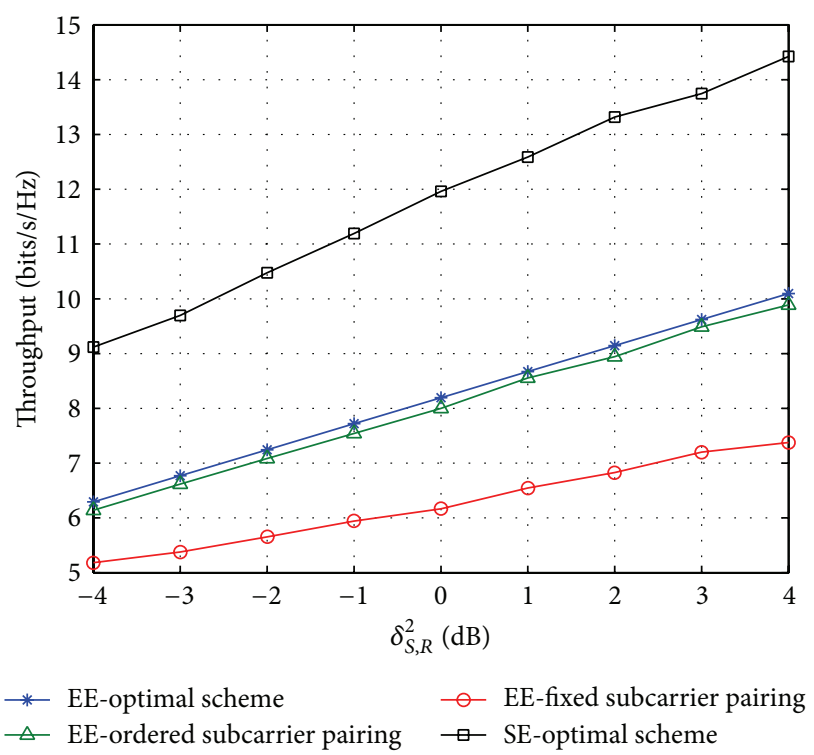

FIgURE 7: Throughput versus channel variance of $S \rightarrow R$ link with $N=12$ relays, and $w_{1}=w_{2}=1$.

Such additional circuit energy consumption will increase with the number of relays. We consider the throughput per unit power rather than the throughput. Therefore, EE will decrease when the energy consumption is greater than its contribution in rate. This phenomenon will occur especially when the number of relays is large.

Figures 5, 6, and 7 plot the throughput corresponding to the EE in Figures 2, 3, and 4, respectively. From them, the throughput of the spectral-efficient design is, as expected, more than that of the energy-efficient design. Combining Figures 2 and 5, Figures 3 and 6, and Figures 4 and 7, we can get that the maximum $\mathrm{EE}$ and the maximum throughput are

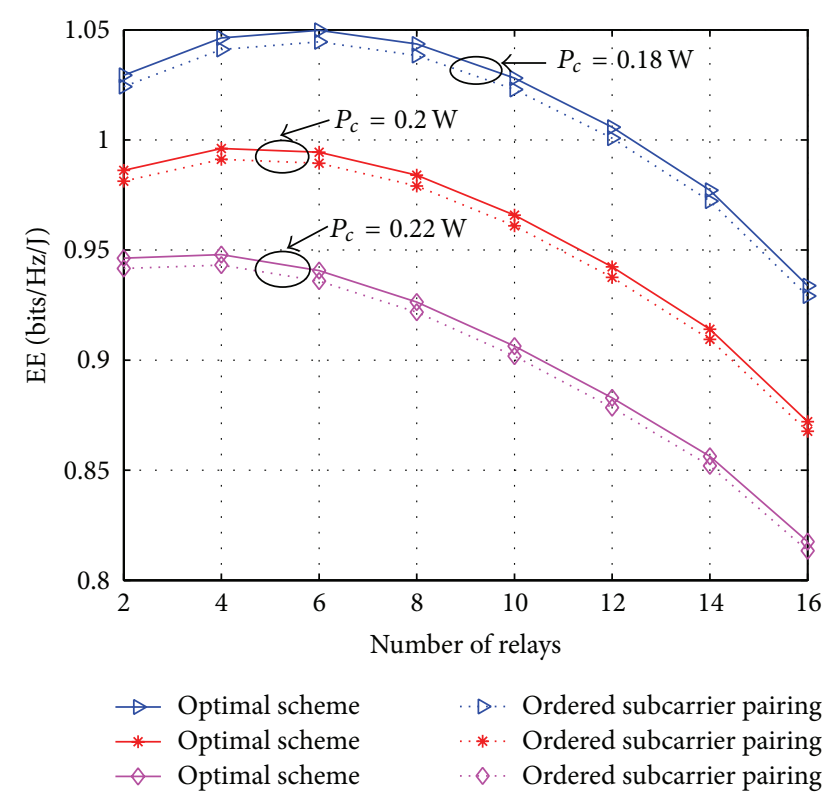

FIGURE 8: Energy efficiency versus different numbers of relays with $w_{1}=w_{2}=1$.

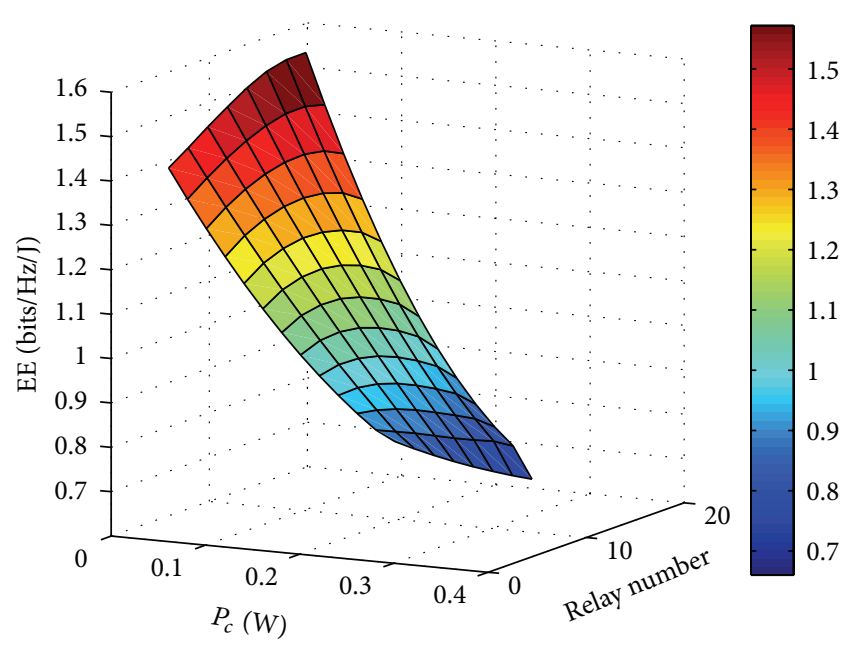

FIGURE 9: Energy efficiency versus different numbers of relays and circuit energy consumption with $w_{1}=w_{2}=1$.

not necessarily simultaneously achieved. Due to the limited space, we will investigate the tradeoff relationship in detail in our future work.

Figure 8 illustrates EE versus different numbers of relays under the proposed optimal scheme and suboptimal schemebased OSP. $\delta_{k, n}^{2}$ and $\delta_{n, D}^{2}$ are set to zero. Figure 8 indicates that, as the relay number increases, EE first increases and then decreases. For different circuit energy consumption, the relay number also differs when $\mathrm{EE}$ reaches the maximum. For example, for $P_{c}=0.18 \mathrm{~W}$, EE reaches the maximum when the number of relays is 6 . However, for $P_{c}=0.2 \mathrm{~W}$, the best relay number is 4 . With the decreased circuit energy consumption, the best relay number increases. To express the relationship clearly, we plot EE versus different relay 
numbers and circuit energy consumption with the optimal scheme only in Figure 9. The figure illustrates that the change of EE with the relay number differs under different circuit energy consumption. For example, when the circuit energy consumption is low, EE increases with the relay number. However, with increasing circuit energy consumption, EE first increases and then decreases. When the circuit energy consumption is high, EE monotone decreases. Therefore, high circuit energy consumption is unfavorable to EE when the number of relays is large.

\section{Conclusion}

In this paper, we formulate an energy-efficient resource allocation problem for a cooperative multirelay OFDMA uplink system with AF protocol. This paper determines the joint optimization of subcarrier pairing, relay assignment, subcarrier assignment, and power allocation with the objective of maximizing EE. Individual power constraint for every user and relay is applied. To solve the complex fraction problem, we transform the considered EE problem in fractional form into an equivalent optimal problem in subtractive form. However, the mixed integer programming problem is NPhard. We utilize the dual method to solve the problem efficiently. To reduce the complexity of the problem, we propose two low-complexity schemes for subcarrier pairing. The simulation results show greater EE improvement of the energy-efficient design than that of the spectral-efficient design, and the performance of the proposed suboptimal algorithm OSP-based is close to that of the optimal algorithm. EE differs under different relay numbers and is also affected by circuit energy consumption. In future work, we will study the fundamental tradeoff between EE and SE in cooperative communication.

\section{Appendices}

\section{A. Proof of Convex Function}

We define the function as

$$
f(x, y)=\log \left(1+x+\frac{x y}{x+y}\right), \quad x, y>0 \text {. }
$$

The Hessian of $f(x, y)$ can be written as

$$
\nabla^{2} f(x, y)=\left[\begin{array}{ll}
\frac{\partial^{2} f}{\partial x^{2}} & \frac{\partial^{2} f}{\partial y \partial x} \\
\frac{\partial^{2} f}{\partial x \partial y} & \frac{\partial^{2} f}{\partial y^{2}}
\end{array}\right]
$$

where

$$
\begin{aligned}
& \frac{\partial^{2} f}{\partial x^{2}}=\frac{-(x+y)^{4}-y^{2}\left[3(x+y)^{2}+2 x+2 y+x^{2}+2 x y\right]}{(x+y)^{2}\left(x+y+x^{2}+2 x y\right)^{2}}, \\
& \frac{\partial^{2} f}{\partial y \partial x}=-\frac{-x^{2}\left[(x+y)^{2}+y^{2}\right]+2 x^{2} y(1+x+y+2 y)}{(x+y)^{2}\left(x+y+x^{2}+2 x y\right)^{2}}, \\
& \frac{\partial^{2} f}{\partial x \partial y}=-\frac{-x^{2}\left[(x+y)^{2}+y^{2}\right]+2 x^{2} y(1+x+y+2 y)}{(x+y)^{2}\left(x+y+x^{2}+2 x y\right)^{2}} \\
& \frac{\partial^{2} f}{\partial y^{2}}=\frac{-x^{2}\left[3 x^{2}+2 x+2 y+4 x y\right]}{(x+y)^{2}\left(x+y+x^{2}+2 x y\right)^{2}}, \\
& =\left(-\left[a(x+y)^{2}+b x^{2}\right]^{2}-\left(a y^{2}+b x^{2}\right)^{2}-2(x+y+2 x y)\right. \\
& \left.\times(a y-b x)^{2}-\left(2 a x y-b x^{2}\right)^{2}-2 a^{2} y^{3}(y+2 x)\right) \\
& \times\left((x+y)^{2}\left(x+y+x^{2}+2 x y\right)^{2}\right)^{-1} \leq 0 .
\end{aligned}
$$

For any $a$ and $b,($ A.7) means that the Hessian of $f(x, y)$ is negative semidefinite, and $f(x, y)$ is jointly concave on $x$ and $y$ [23]. Using affine mapping operation, $f(\alpha x, \beta y)$ remains the concavity on $x$ and $y$, where $\alpha$ and $\beta$ are constant values.

\section{B. Proof of Theorems 1-3}

Proof of Theorem 1. We assume that $q_{1}>q_{2}$ and $F\left(q_{1}\right)=$ $R\left(\boldsymbol{\rho}_{1}, \boldsymbol{\gamma}_{1}, \mathbf{p}_{1}\right)-q_{1}\left(\boldsymbol{\rho}_{1}, \boldsymbol{\gamma}_{1}, \mathbf{p}_{1}\right)$. Consider

$$
\begin{aligned}
F\left(q_{1}\right) & =\max _{\boldsymbol{\rho}, \boldsymbol{\gamma}, \mathbf{p} \in \boldsymbol{\pi}}\left\{R(\boldsymbol{\rho}, \boldsymbol{\gamma}, \mathbf{p})-q_{1}(\boldsymbol{\rho}, \boldsymbol{\gamma}, \mathbf{p})\right\} \\
& =R\left(\boldsymbol{\rho}_{1}, \boldsymbol{\gamma}_{1}, \mathbf{p}_{1}\right)-q_{1}\left(\boldsymbol{\rho}_{1}, \boldsymbol{\gamma}_{1}, \mathbf{p}_{1}\right) \\
& <R\left(\boldsymbol{\rho}_{1}, \boldsymbol{\gamma}_{1}, \mathbf{p}_{1}\right)-q_{2}\left(\boldsymbol{\rho}_{1}, \boldsymbol{\gamma}_{1}, \mathbf{p}_{1}\right) \\
& \leq \max _{\boldsymbol{\rho}, \boldsymbol{\gamma}, \mathbf{p} \in \boldsymbol{\pi}}\left\{R(\boldsymbol{\rho}, \boldsymbol{\gamma}, \mathbf{p})-q_{2}(\boldsymbol{\rho}, \boldsymbol{\gamma}, \mathbf{p})\right\}=F\left(q_{2}\right) .
\end{aligned}
$$

Proof of Theorem 2. Considering the integer variables $\rho_{k, n}^{m, m^{\prime}}$ and $\gamma_{m, m^{\prime}}$, the feasible domain of $(\boldsymbol{\rho}, \boldsymbol{\gamma})$ is a discrete and finite set that consists of all possible subcarrier allocations. Therefore, $F(q)$ is generally a continuous but nondifferentiable function for $q$. Given that $\lim _{q \rightarrow-\infty} F(q)=+\infty$ and $\lim _{q \rightarrow \infty} F(q)=-\infty$, as well as considering Theorem 1, we can obtain Theorem 2 . 
Proof of Theorem 3. We define problem 1 as follows:

$$
q_{0}=\frac{R\left(\boldsymbol{\rho}_{0}, \boldsymbol{\gamma}_{0}, \mathbf{p}_{0}\right)}{P\left(\boldsymbol{\rho}_{0}, \boldsymbol{\gamma}_{0}, \mathbf{p}_{0}\right)}=\max _{\boldsymbol{\rho}, \boldsymbol{\gamma}, \mathbf{p} \in \pi} \frac{R(\boldsymbol{\rho}, \boldsymbol{\gamma}, \mathbf{p})}{P(\boldsymbol{\rho}, \boldsymbol{\gamma}, \mathbf{p})}
$$

Problem 2 is expressed as follows:

$$
\begin{aligned}
F\left(q_{0}\right) & =F\left(q_{0},\left(\boldsymbol{\rho}_{0}, \boldsymbol{\gamma}_{0}, \mathbf{p}_{0}\right)\right) \\
& =\max _{\boldsymbol{\rho}, \boldsymbol{\gamma}, \mathbf{p} \in \boldsymbol{\pi}}\left\{R(\boldsymbol{\rho}, \boldsymbol{\gamma}, \mathbf{p})-q_{0}(\boldsymbol{\rho}, \boldsymbol{\gamma}, \mathbf{p})\right\}=0 .
\end{aligned}
$$

(a) If we have known

$$
\begin{aligned}
q_{0} & =\frac{R\left(\boldsymbol{\rho}_{0}, \boldsymbol{\gamma}_{0}, \mathbf{p}_{0}\right)}{P\left(\boldsymbol{\rho}_{0}, \boldsymbol{\gamma}_{0}, \mathbf{p}_{0}\right)} \\
& =\max _{\boldsymbol{\rho}, \boldsymbol{\gamma}, \mathbf{p} \in \pi} \frac{R(\boldsymbol{\rho}, \boldsymbol{\gamma}, \mathbf{p})}{P(\boldsymbol{\rho}, \boldsymbol{\gamma}, \mathbf{p})} \geq \frac{R(\boldsymbol{\rho}, \boldsymbol{\gamma}, \mathbf{p})}{P(\boldsymbol{\rho}, \boldsymbol{\gamma}, \mathbf{p})},
\end{aligned}
$$

we can obtain

$$
\begin{gathered}
R(\boldsymbol{\rho}, \boldsymbol{\gamma}, \mathbf{p})-q_{0} P(\boldsymbol{\rho}, \boldsymbol{\gamma}, \mathbf{p}) \leq 0 \\
R\left(\boldsymbol{\rho}_{0}, \boldsymbol{\gamma}_{0}, \mathbf{p}_{0}\right)-q_{0} P\left(\boldsymbol{\rho}_{0}, \boldsymbol{\gamma}_{0}, \mathbf{p}_{0}\right)=0
\end{gathered}
$$

According to (B.5a), we have $F\left(q_{0}\right)$ = $\max _{\boldsymbol{\rho}, \boldsymbol{\gamma}, \mathbf{p} \in \boldsymbol{\pi}}\left\{R(\boldsymbol{\rho}, \boldsymbol{\gamma}, \mathbf{p})-q_{0} P(\boldsymbol{\rho}, \boldsymbol{\gamma}, \mathbf{p})\right\}=0$. Equation (B.5b) indicates that the maximum is $\left(\boldsymbol{\rho}_{0}, \gamma_{0}, \mathbf{p}_{0}\right)$. Thus, the first part of the proof is finished.

(b) If we know

$$
\begin{aligned}
F\left(q_{0}\right) & =F\left(q_{0},\left(\boldsymbol{\rho}_{0}, \boldsymbol{\gamma}_{0}, \mathbf{p}_{0}\right)\right) \\
& =\max _{\boldsymbol{\rho}, \boldsymbol{\gamma}, \mathbf{p} \in \boldsymbol{\pi}}\left\{R(\boldsymbol{\rho}, \boldsymbol{\gamma}, \mathbf{p})-q_{0}(\boldsymbol{\rho}, \boldsymbol{\gamma}, \mathbf{p})\right\}=0,
\end{aligned}
$$

then we can have

$$
\begin{aligned}
& R(\boldsymbol{\rho}, \boldsymbol{\gamma}, \mathbf{p})-q_{0} P(\boldsymbol{\rho}, \boldsymbol{\gamma}, \mathbf{p}) \\
& \quad \leq R\left(\boldsymbol{\rho}_{0}, \boldsymbol{\gamma}_{0}, \mathbf{p}_{0}\right)-q_{0} P\left(\boldsymbol{\rho}_{0}, \boldsymbol{\gamma}_{0}, \mathbf{p}_{0}\right)=0
\end{aligned}
$$

Hence,

$$
\begin{array}{r}
R(\boldsymbol{\rho}, \boldsymbol{\gamma}, \mathbf{p})-q_{0} P(\boldsymbol{\rho}, \boldsymbol{\gamma}, \mathbf{p}) \leq 0 \\
R\left(\boldsymbol{\rho}_{0}, \boldsymbol{\gamma}_{0}, \mathbf{p}_{0}\right)-q_{0} P\left(\boldsymbol{\rho}_{0}, \boldsymbol{\gamma}_{0}, \mathbf{p}_{0}\right)=0
\end{array}
$$

Using (B.8a), we have $q_{0} \geq R(\boldsymbol{\rho}, \boldsymbol{\gamma}, \mathbf{p}) / P(\boldsymbol{\rho}, \boldsymbol{\gamma}, \mathbf{p})$. Thus, $q_{0}$ is the maximum of problem 1 . With $(\mathrm{B} .8 \mathrm{~b})$, we have $q_{0}=$ $R\left(\boldsymbol{\rho}_{0}, \boldsymbol{\gamma}_{0}, \mathbf{p}_{0}\right) / P\left(\boldsymbol{\rho}_{0}, \boldsymbol{\gamma}_{0}, \mathbf{p}_{0}\right)$. Therefore, $\left(\boldsymbol{\rho}_{0}, \boldsymbol{\gamma}_{0}, \mathbf{p}_{0}\right)$ is a solution vector of problem 1 .

\section{Derivation of $(24)$}

We present the detailed derivation about (24) in (C.1) as follows:

$$
\begin{aligned}
& g(\lambda, \mu, v) \\
& \triangleq \max _{\{\boldsymbol{\rho}, \boldsymbol{\gamma}\} \in \boldsymbol{\Omega}} L(\boldsymbol{\rho}, \boldsymbol{\gamma}, \mathbf{p}, \boldsymbol{\lambda}, \boldsymbol{\mu}, \boldsymbol{v}) \\
& =\max _{\{\boldsymbol{\rho}, \boldsymbol{\gamma}\} \in \Omega} \sum_{k=1}^{K} \sum_{n=1}^{N} \sum_{m=1}^{M} \sum_{m^{\prime}=1}^{M} w_{k} r_{k, n}^{m, m^{\prime}} \\
& -q\left(\xi \sum_{k=1}^{K} \sum_{n=1}^{N} \sum_{m=1}^{M} \sum_{m^{\prime}=1}^{M}\left(p_{S, k}^{m}+p_{R, n}^{m^{\prime}}\right)+\sum_{k=1}^{K} P_{C, S}^{k}+\sum_{n=1}^{N} P_{C, R}^{n}\right) \\
& +\sum_{k=1}^{k} \lambda_{k}\left(P_{S, k}-\sum_{m=1}^{M} p_{S, k}^{m}\right) \\
& +\sum_{n=1}^{N} \mu_{n}\left(P_{S, n}-\sum_{m^{\prime}=1}^{M} p_{R, n}^{m^{\prime}}\right)+\sum_{k=1}^{k} v_{k}\left(R_{S, k}-Q_{S, k}\right) \\
& =\max _{\{\boldsymbol{\rho}, \boldsymbol{\gamma}\} \in \boldsymbol{\Omega}} \sum_{k=1}^{K} \sum_{n=1}^{N} \sum_{m=1}^{M} \sum_{m^{\prime}=1}^{M} w_{k} r_{k, n}^{m, m^{\prime}}+\sum_{k=1}^{k} v_{k} R_{S, k} \\
& -q \xi \sum_{k=1}^{K} \sum_{n=1}^{N} \sum_{m=1}^{M} \sum_{m^{\prime}}^{M} p_{S, k}^{m}-\sum_{k=1}^{k} \lambda_{k} \sum_{m=1}^{M} p_{S, k}^{m}-q \xi \sum_{k=1}^{K} \sum_{n=1}^{N} \sum_{m=1}^{M} \sum_{m^{\prime}=1}^{M} p_{R, n}^{m^{\prime}} \\
& -\sum_{n=1}^{N} \mu_{n} P_{S, n}+\sum_{k=1}^{k} \lambda_{k} P_{S, k}+\sum_{n=1}^{N} \mu_{n} P_{S, n} \\
& +q\left(\sum_{k=1}^{K} P_{C, S}^{k}+\sum_{n=1}^{N} P_{C, R}^{n}\right)-\sum_{k=1}^{k} v_{k} Q_{S, k} \\
& =\max _{\{\boldsymbol{\rho}, \boldsymbol{\gamma}\} \in \boldsymbol{\Omega}} \sum_{k=1}^{K} \sum_{n=1}^{N} \sum_{m=1}^{M} \sum_{m^{\prime}=1}^{M}\left(w_{k}\left(1+v_{k}\right) r_{k, n}^{m, m^{\prime}}-\left(q \xi+\lambda_{k}\right) p_{S, k}^{m}\right. \\
& \left.-\left(q \xi+\mu_{n}\right) p_{R, n}^{m^{\prime}}\right) \\
& +\sum_{k=1}^{k} \lambda_{k} P_{S, k}+\sum_{n=1}^{N} \mu_{n} P_{S, n}+q\left(\sum_{k=1}^{K} P_{C, S}^{k}+\sum_{n=1}^{N} P_{C, R}^{n}\right)-\sum_{k=1}^{k} v_{k} Q_{S, k} .
\end{aligned}
$$

\section{Solving Process of (26)}

We can obtain the partial derivative of $L$ below. 
For simplicity, variables $p_{S, k}^{m}, p_{R, n}^{m^{\prime}}, \alpha_{k, D}^{m}, \alpha_{k, n}^{m}$, and $\alpha_{n, D}^{m^{\prime}}$ are replaced by $P_{1}, P_{2}, \alpha_{1}, \alpha_{2}$, and $\alpha_{3}$, respectively. Consider

$$
\frac{\partial L}{\partial p_{1}}=\frac{w(1+v)}{2 \ln 2} \cdot \frac{1}{a} \cdot \frac{\alpha_{3}\left(p_{1} \alpha_{1}+p_{2} \alpha_{2}\right)^{2}+p_{2}^{2} \alpha_{1} \alpha_{2}^{2}}{p_{1} \alpha_{1}+p_{2} \alpha_{2}}-(q \xi+\lambda)
$$

$$
\begin{aligned}
\frac{\partial L}{\partial p_{2}}= & \frac{w(1+v)}{2 \ln 2} \cdot \frac{1}{a} \cdot \frac{p_{1}^{2} \alpha_{1}^{2} \alpha_{2}}{p_{1} \alpha_{1}+p_{2} \alpha_{2}}-(q \xi+\mu) \\
a= & p_{1} \alpha_{1}+p_{2} \alpha_{2} \\
& +p_{1}\left(p_{1} \alpha_{1} \alpha_{3}+p_{2} \alpha_{2} \alpha_{3}+p_{2} \alpha_{1} \alpha_{2}\right) .
\end{aligned}
$$

When $P_{1}$ and $P_{2}$ are positive, (D.1) and (D.2) will be zero. Thus, we can have

$$
\begin{aligned}
(q \xi+\mu) & {\left[\alpha_{3}\left(p_{1} \alpha_{1}+p_{2} \alpha_{2}\right)^{2}+p_{2}^{2} \alpha_{1} \alpha_{2}^{2}\right] } \\
& =(q \xi+\lambda)\left(p_{1}^{2} \alpha_{1}^{2} \alpha_{2}\right) .
\end{aligned}
$$

Equation (D.3) can be simplified as follows:

$$
p_{1}=b p_{2}
$$

where

$$
\begin{aligned}
b= & \left(\alpha _ { 2 } \left(\left(( q \xi + \mu ) \left(\alpha_{1} \alpha_{2}(q \xi+\lambda)-\alpha_{1} \alpha_{3}(q \xi+\mu)\right.\right.\right.\right. \\
& \left.\left.\left.\left.+\alpha_{2} \alpha_{3}(q \xi+\lambda)\right)\right)^{1 / 2}+\alpha_{3}(q \xi+\mu)\right)\right) \\
& \times\left(\alpha_{1}\left[\alpha_{2}(q \xi+\lambda)-\alpha_{3}(q \xi+\mu)\right]\right)^{-1} .
\end{aligned}
$$

The factor $b$ must be greater than zero. We therefore have $\alpha_{2}(q \xi+\lambda)>\alpha_{3}(q \xi+\mu)$. Substituting (D.4) into (D.1), we obtain

$$
\begin{aligned}
p_{2}= & \frac{w(1+v)}{2 \ln 2} \\
& \times\left(\alpha_{1} \alpha_{2}^{2}+\left(\alpha_{3}-\frac{2(q \xi+\lambda) \ln 2}{w(1+v)}\right)\left(\alpha_{1} b+\alpha_{2}^{2}\right)^{2}\right) \\
& \times\left(b(q \xi+\lambda)\left(\alpha_{1} b+\alpha_{2}\right)\left(\alpha_{3} \alpha_{1} b+\alpha_{2} \alpha_{3}+\alpha_{1} \alpha_{2}\right)\right)^{-1}
\end{aligned}
$$

If $p_{2}<0$, then $P_{2}$ should be set to zero.

When $\alpha_{2}(q \xi+\lambda) \leq \alpha_{3}(q \xi+\mu), p_{2}=0$. For these cases, the optimal power allocation in the first phase can be expressed as follows:

$$
p_{1}=\left(\frac{w(1+v)}{2 \ln 2(q+\lambda)}-\frac{1}{\alpha_{3}}\right)^{+}
$$

Thus, (27) and (28) are proved.

\section{Conflict of Interests}

The authors declare that there is no conflict of interests regarding the publication of this paper.

\section{Acknowledgment}

This study is funded by the National Natural Science Foundation of China (Grant no. 61271421).

\section{References}

[1] A. Nosratinia, T. E. Hunter, and A. Hedayat, "Cooperative communication in wireless networks," IEEE Communications Magazine, vol. 42, no. 10, pp. 74-80, 2004.

[2] Ö. Oyman, J. N. Laneman, and S. Sandhu, "Multihop relaying for broadband wireless mesh networks: from theory to practice," IEEE Communications Magazine, vol. 45, no. 11, pp. 116-122, 2007.

[3] W. Su, A. K. Sadek, and K. J. Ray Liu, "Cooperative communication protocols in wireless networks: performance analysis and optimum power allocation," Wireless Personal Communications, vol. 44, no. 2, pp. 181-217, 2008.

[4] J. N. Laneman, D. N. C. Tse, and G. W. Wornell, "Cooperative diversity in wireless networks: efficient protocols and outage behavior," IEEE Transactions on Information Theory, vol. 50, no. 12, pp. 3062-3080, 2004.

[5] D. W. K. Ng, E. S. Lo, and R. Schober, "Energy-efficient resource allocation in multi-cell OFDMA systems with limited backhaul capacity," in Proceedings of the IEEE Wireless Communications and Networking Conference (WCNC '12), Paris, France, 2012.

[6] Y. Li, W. Wang, J. Kong, and M. Peng, "Subcarrier pairing for amplify-and-forward and decode-and-forward OFDM relay links," IEEE Communications Letters, vol. 13, no. 4, pp. 209-211, 2009.

[7] Y. Li, W. Wang, J. Kong, W. Hong, X. Zhang, and M. Peng, "Power allocation and subcarrier pairing in OFDM-based relaying networks," in Proceedings of the IEEE International Conference on Communications (ICC '08), pp. 2602-2606, May 2008.

[8] M. Kaneko, K. Hayashi, P. Popovski, K. Ikeda, H. Sakai, and R. Prasad, "Amplify-and-forward cooperative diversity schemes for Multi-Carrier systems," IEEE Transactions on Wireless Communications, vol. 7, no. 5, pp. 1845-1850, 2008.

[9] W. Dang, M. Tao, H. Mu, and J. Huang, "Subcarrier-pair based resource allocation for cooperative multi-relay OFDM systems," IEEE Transactions on Wireless Communications, vol. 9, no. 5, pp. 1640-1649, 2010.

[10] M. S. Alam, J. W. Mark, and X. S. Shen, "Relay selection and resource allocation for multi-user cooperative OFDMA networks," IEEE Transactions on Wireless Communications, vol. 12, no. 5, pp. 2193-2205, 2013.

[11] X. Zhang, W. Jiao, and M. Tao, "End-to-end resource allocation in OFDM based linear multi-hop networks," in Proceedings of the 27th IEEE Communications Society Conference on Computer Communications (INFOCOM '08), Phoenix, Ariz, USA, April 2008.

[12] X. Zhang, M. Tao, W. Jiao, and C. S. Ng, "End-to-end outage minimization in OFDM based linear relay networks," IEEE Transactions on Communications, vol. 57, no. 10, pp. 3034-3044, 2009.

[13] C. N. Hsu, H. J. Su, and P. H. Lin, “Joint subcarrier pairing and power allocation for OFDM transmission with decode-andforward relaying," IEEE Transactions on Signal Processing, vol. 59, no. 1, pp. 399-414, 2011. 
[14] D. W. K. Ng and R. Schober, "Cross-layer scheduling for OFDMA amplify-and-forward relay networks," IEEE Transactions on Vehicular Technology, vol. 59, no. 3, pp. 1443-1458, 2010.

[15] Y. Chen, S. Zhang, S. Xu, and G. Y. Li, "Fundamental trade-offs on green wireless networks," IEEE Communications Magazine, vol. 49 , no. 6 , pp. 30-37, 2011.

[16] T. Chen, H. Kim, and Y. Yang, "Energy efficiency metrics for green wireless communications," in Proceedings of the International Conference on Wireless Communications and Signal Processing (WCSP '10), pp. 1-6, October 2010.

[17] C. Han, T. Harrold, S. Armour et al., "Green radio: radio techniques to enable energy-efficient wireless networks," IEEE Communications Magazine, vol. 49, no. 6, pp. 46-54, 2011.

[18] H. Bogucka and A. Conti, "Degrees of freedom for energy savings in practical adaptive wireless systems," IEEE Communications Magazine, vol. 49, no. 6, pp. 38-45, 2011.

[19] C. Xiong, G. Y. Li, S. Zhang, Y. Chen, and S. Xu, "Energyefficient resource allocation in OFDMA networks," in Proceedings of the 54th Annual IEEE Global Telecommunications Conference (GLOBECOM '11), pp. 1-5, Houston, Tex, USA, December 2011.

[20] C. Xiong, G. Y. Li, S. Zhang, Y. Chen, and S. Xu, "Energy- and spectral-efficiency tradeoff in downlink OFDMA networks," IEEE Transactions on Wireless Communications, vol. 10, no. 11, pp. 3874-3886, 2011.

[21] D. W. K. Ng, E. S. Lo, and R. Schober, "Energy-efficient resource allocation in multi-cell OFDMA systems with limited backhaul capacity," IEEE Transactions on Wireless Communications, vol. 11, no. 10, pp. 3618-3631, 2012.

[22] D. W. K. Ng, E. S. Lo, and R. Schober, "Wireless information and power transfer: energy efficiency optimization in OFDMA systems," IEEE Transactions on Wireless Communications, vol. 12, no. 12, pp. 6352-6370, 2013.

[23] R. S. Prabhu and B. Daneshrad, "An energy-efficient waterfilling algorithm for OFDM systems," in Proceedings of the IEEE International Conference on Communications (ICC '10), May 2010.

[24] X. Xiao, X. Tao, and J. Lu, "QoS-aware energy-efficient radio resource scheduling in multi-user OFDMA systems," IEEE Communications Letters, vol. 17, no. 1, pp. 75-78, 2013.

[25] J. Zhang, Y. Jiang, and X. Li, "Energy-efficient resource allocation in multiuser relay-based OFDMA networks," Concurrency and Computation: Practice and Experience, vol. 25, no. 9, pp. 1113-1125, 2013.

[26] H. Yu, H. Qin, Y. Z. Li, Y. Zhao, and J. Wang, "Energyefficient power allocation for non-regenerative OFDM relay links," Science China Information Sciences, vol. 56, no. 2, pp. 185192, 2013.

[27] J. Tang and X. Zhang, "Cross-layer resource allocation over wireless relay networks for quality of service provisioning," IEEE Journal on Selected Areas in Communications, vol. 25, no. 4, pp. 645-655, 2007.

[28] S. Cui, A. J. Goldsmith, and A. Bahai, "Energy-constrained modulation optimization," IEEE Transactions on Wireless Communications, vol. 4, no. 5, pp. 2349-2360, 2005.

[29] W. Dinkelbach, "On nonlinear fractional programming," Management Science, vol. 13, no. 7, pp. 492-498, 1967.

[30] W. Yu and R. Lui, "Dual methods for nonconvex spectrum optimization of multicarrier systems," IEEE Transactions on Communications, vol. 54, no. 7, pp. 1310-1322, 2006.
[31] S. Boyd and L. Vandenberghe, Convex Optimization, Cambridge University Press, Cambridge, UK, 2004.

[32] S. Boyd, L. Xiao, and A. Mutapcic, "Subgradient methods," lecture Notes EE392o, Stanford University, Stanford, Calif, USA, 2004.

[33] H. W. Kuhn, "The Hungarian method for the assignment problem," Naval Research Logistics Quarterly, vol. 2, pp. 83-97, 1955. 

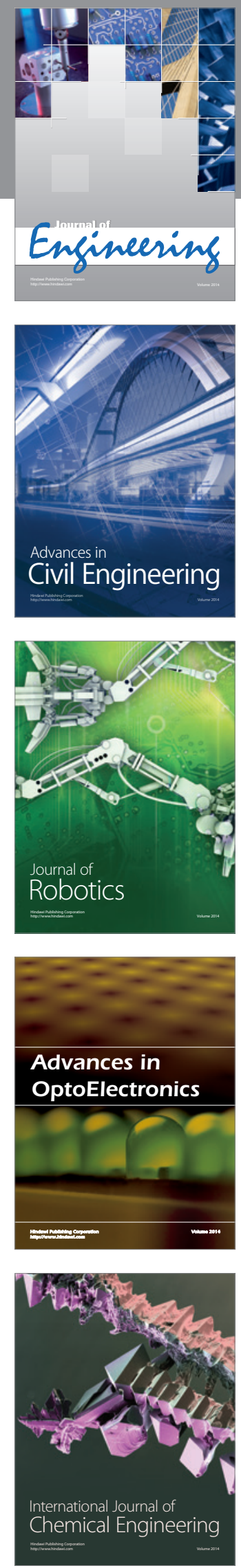

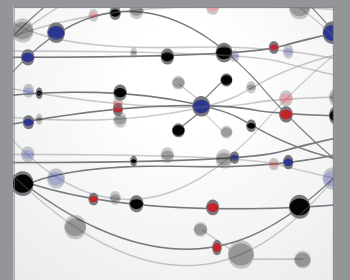

The Scientific World Journal
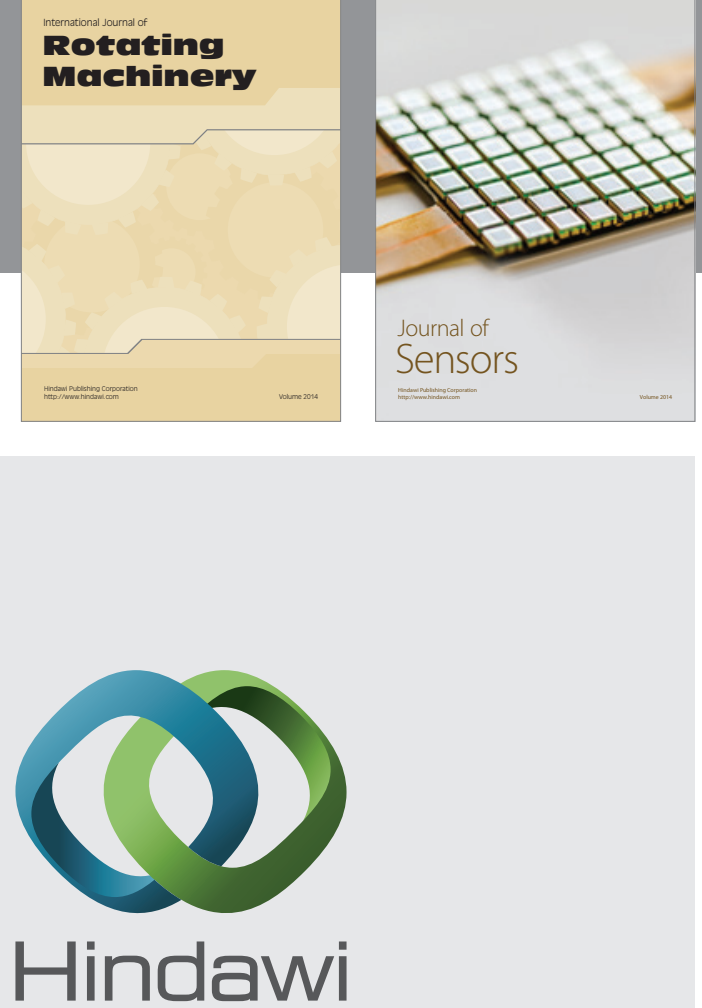

Submit your manuscripts at http://www.hindawi.com
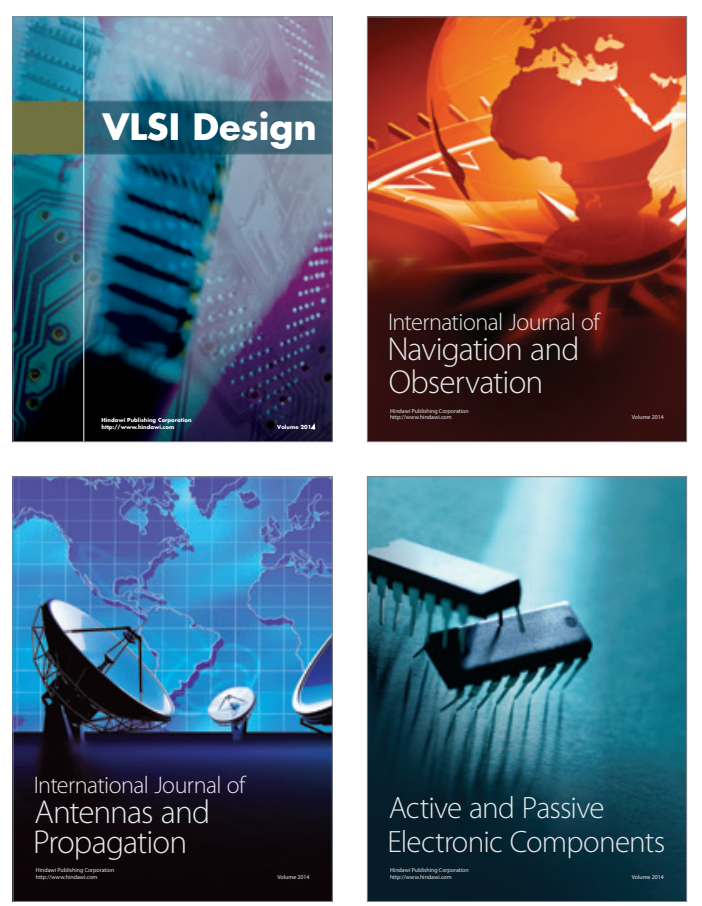
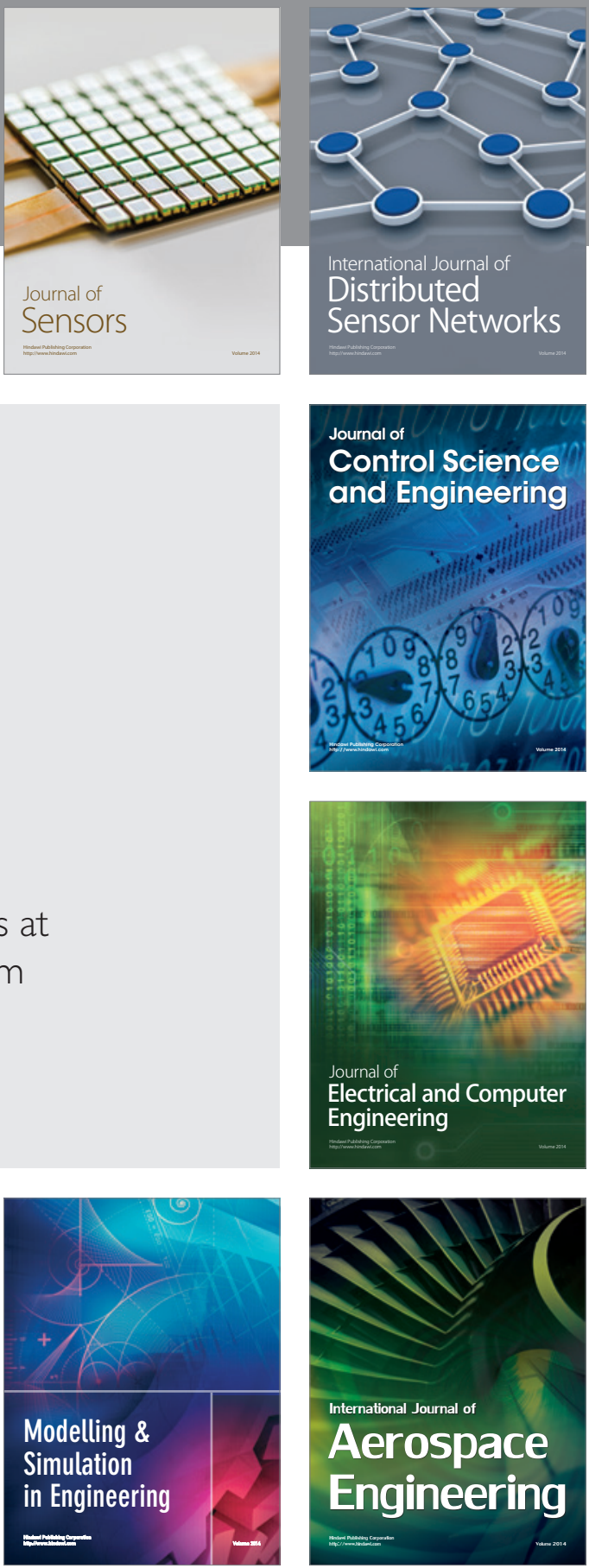

Journal of

Control Science

and Engineering
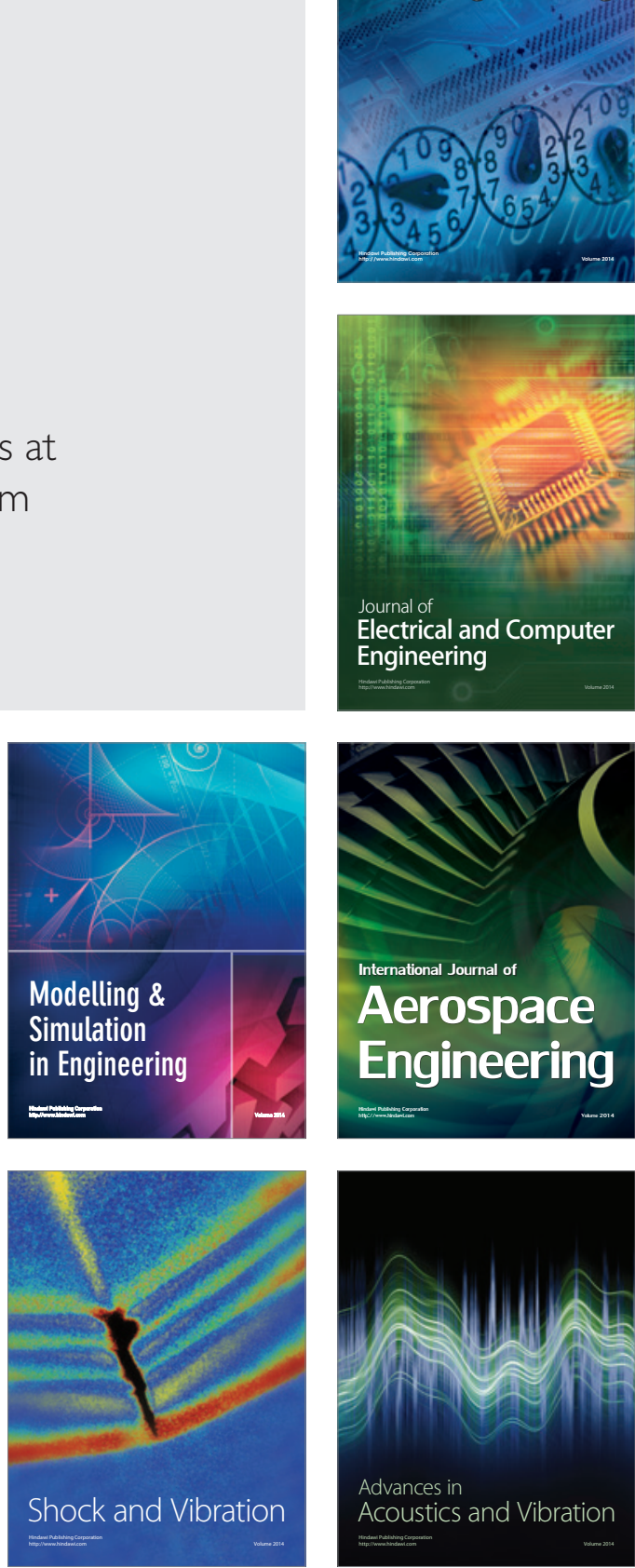